\title{
Prediction of Coronary Artery Disease in Patients Undergoing Operations for Rheumatic Aortic Valve Disease
}

Department of Cardiothoracic Surgery

Changhai Hospital

168 Changhai Road

Shanghai 200433, China

zhiyunx1@163.com

Tao Yan, MD; Guan-xin Zhang, MD; Bai-ling Li, MD; Lin Han, MD; Jia-jie Zang, MD; Li Li, MD; Zhi-yun Xu, MD

Department of Cardiothoracic Surgery (Yan, Zhang, B. Li, Han, L. Li, Xu), Changhai Hospital, Shanghai, China; Department of Health Statistics (Zang), Second Military Medical University, Shanghai, China

Background: We sought to develop and validate a logistic model and a simple score system for prediction of significant coronary artery disease (CAD) in patients undergoing operations for rheumatic aortic valve disease. Hypothesis: The simple score model we established based on the logistic model was efficient and practical. Methods: A total of 669 rheumatic patients (mean age $51 \pm 9$ years), who underwent routine coronary angiography (CAG) before aortic valve surgery between 1998 and 2010, were analyzed. A bootstrap-validated logistic regression model on the basis of clinical risk factors was developed to identify low-risk ( $\leq 5 \%$ ) patients, from which an additive model was derived. Receiver operating characteristic (ROC) curves were used to compare discrimination, and precision was quantified by the Hosmer-Lemeshow statistic. Significant coronary atherosclerosis was defined as 50\% or more luminal narrowing in 1 or more major epicardial vessels determined by means of coronary angiography.

Results: Eighty-eight (13.2\%) patients had significant coronary atherosclerosis. Independent predictors of CAD include age, angina, diabetes mellitus, and hypertension. A total of 325 patients were designated as low risk according to the bootstrap logistic regression and additive models. Of these patients, only 4 (1.2\%) had single-vessel disease, and none had high-risk CAD (ie, left main trunk, proximal left anterior descending, or multivessel disease). The bootstrap logistic regression and additive models show good discrimination, with an area under the ROC curve of 0.948 and 0.942 , respectively.

Conclusions: Our logistic regression model can reliably estimate the prevalence of significant CAD in rheumatic patients undergoing aortic valve operation, while the additive simple score system could reliably identify the low-risk patients in whom routine preoperative angiography might be safely avoided.

\section{Introduction}

Coronary artery disease (CAD) has a detrimental effect on long-term survival in patients undergoing aortic valve operations. ${ }^{1}$ It is of great importance to detect the presence of coexistent $\mathrm{CAD}$ before aortic valve operations. It is impractical for every patient to undergo coronary angiography because of the low positive rate, the potential complications, and the cost. There is no validated approach and model for routine evaluation of possible $\mathrm{CAD}$ in these patients. The current American College of Cardiology/American Heart Association (AHA/ACC) practice guidelines ${ }^{2}$ for presurgical coronary artery angiography (CAG) include the following: male patients 35 years of age or older; female patients who are postmenopausal or premenopausal and

The authors have no funding, financial relationships, or conflicts of interest to disclose.

Dr. Guan-xin Zhang and Dr. Bai-ling Li have contributed equally to the work. Dr. Lin-han is co-corresponding author (sh_hanlin@hotmail.com).
35 years of age or older with coronary risk factors; and patients with chest pain, objective evidence of ischemia, 1 or more risk factors for $\mathrm{CAD}$, previous $\mathrm{CAD}$, or decreased left ventricular (LV) systolic function. However, the patients with aortic valve disease in China and many other developing countries were different from that of in the United States and other developed countries. For the former, the prevalent is rheumatic valve disease, and the latter, degenerative valve disease. In these patients, the risk of coexistent $\mathrm{CAD}$ is much lower ${ }^{3,4}$ and therefore the guideline might not be applicable for prediction. In this work, we sought to develop a new model to predict the risk of CAD in patients undergoing operation for rheumatic aortic valve disease.

\section{Methods}

The study was performed with protocols approved by the Ethics Committee in Research of Changhai Hospital.

\section{Patient Selection}

We retrospectively identified 902 consecutive patients from the Changhai Hospital who underwent operations for 
rheumatic aortic valve disease between 1998 and 2010 . We excluded the patients who were diagnosed as CAD patients because of previous myocardial infraction. Among those patients, 207 did not undergo CAG at the discretion of the attending cardiologist and 26 patients with previous myocardial infraction were excluded. Thus, 669 patients were included in our study. Routine CAG was performed before valve surgery. The presence of significant coronary disease was defined as luminal narrowing of at least $50 \%$ of the diameter of a major coronary artery.

\section{Risk Factor Selection}

Risk factors for $\mathrm{CAD}$ were defined as follows: age, sex, family history of CAD (first-degree relative with a myocardial infarction before age of 50 years in men and before 60 years in women), smoking, diabetes mellitus, hypertension, hypercholesterolemia (defined as receiving medication for hypercholesterolemia or serum cholesterol of $\geq 5.8 \mathrm{mmol} / \mathrm{L}$ ). In addition, the presence of ischemia changes on electrocardiogram (EGC) (defined as any resting ST-segment or T-wave abnormality), coexistent mitral valve lesion, abnormal LV function (defined as an ejection fraction of $50 \%$ or less by means of echocardiography or contrast ventriculography), C-reactive protein (CRP, defined as serum CRP $\geq 3.0 \mathrm{mmol} / \mathrm{L}$ ), and New York Heart Association class IIIIV were also designated as variables for analyses. Etiology was determined by echocardiography and histopathologic examinations.

\section{Statistical Analysis}

Logistic Regression Model Development: All the designate variables were entered into univariable analyses. The criterion for variable retention was significance of 0.2 . Predictors of significant $\mathrm{CAD}$ in the univariable analyses were entered into a forward stepwise multivariable logistic regression model. Only independent predictors $(P<0.05)$ were included in the model:

$$
\ln O R=\alpha+\chi_{1} \beta_{1}+\chi_{2} \beta_{2}+\chi_{3} \beta_{3}+\chi_{4} \beta_{4}+\ldots+\chi_{\mathrm{k}} \beta_{\mathrm{k}}
$$

(ln $O R$ is the natural logarithm of the odds ratio; $\alpha$ is a constant; $\chi_{1} \ldots$, and $\chi_{k}$ are independent predictors; $\beta_{1} \ldots$, and $\beta_{\mathrm{k}}$ represent respective parameter coefficients)

$$
\text { Risk of significant } \mathrm{CAD}=\mathrm{e}^{\ln O R} /\left(\mathrm{e}^{\ln O R}+1\right)
$$

The multivariable logistic model was validated and further refined by using the bootstrap technique, ${ }^{5-7}$ which used 1000 random resamplings to evaluate the stability of the odds ratio estimated with sampling variation.

Simple Additive Score Model: To simplify the calculation process of clinical risks and help the clinician assess the patients much more easily, a simple additive score model was established based on the bootstrap logistic regression model. We choose a probability of significant CAD of greater than 0.05 as a cutoff, which corresponded to a bootstrap logistic regression model score of -2.8 .

$$
\begin{aligned}
& 0.8 \times \text { interval age }^{*}+6.7 \times \text { angina }+1.5 \\
& \times \text { diabetes mellitus }+1.2 \times \text { hypertension }-6.9>-2.8
\end{aligned}
$$

*Interval age $=($ age -35$) / 5$; the youngest of our study population is 35 years old.

By multiplying each coefficient of the equation by 2 , and rounding off the new coefficient to the nearest integer, a simple additive score model was established as follows:

$$
\begin{aligned}
& 2 \times \text { interval age }+13 \times \text { angina }+3 \times \text { diabites mellitus } \\
& +2 \times \text { hypertension }>8
\end{aligned}
$$

Comparing the Logistic Model with the Simple Additive Model: Model discrimination was examined by constructing receiver operating characteristic (ROC) curves for the logistic model and simple additive model. The HosmerLemeshow goodness-of-fit statistic was used to describe the precision of the models.

Continuous variables are expressed as mean values \pm standard deviation (SD), Categorical variables are expressed as frequencies, percentages, or both. All statistical analyses were performed with PASW statistics 18 software.

\section{Results \\ Characteristics of the Study Population}

Clinical characteristics of the study population are summarized in Table 1. Of these 669 patients, 88 (13.2\%) had significant CAD, and 77 (11.5\%) received concomitant coronary artery bypass graft surgery (CABG).

\section{Logistic Regression Model}

The univariable association between significant $\mathrm{CAD}$ and the risk factors evaluated as potential predictors are shown in Table 2. Family history, smoking, abnormal LV function, and ischemic changes on ECG did not reach the significance cutoff 0.2 for variable retention. Age, angina, diabetes mellitus, and hypertension were predictors in the logistic multivariable analysis, and further validated as predictive in the bootstrap-refined model as follows:

$$
\begin{aligned}
& \text { Model score }(\ln O R)=0.823 \times \text { interval age }+6.56 \\
& \times \text { angina }+1.466 \times \text { diabetes mellitus }+1.227 \\
& \times \text { hypertension }-6.896
\end{aligned}
$$

The comparisons between the original logistic regression model and the bootstrap logistic model on the odds ratio with confidence intervals are shown in Table 3 . The odds ratios of the 2 models were of a high degree of similarity, and this confirmed the stability and validity of the model.

\section{Simple Additive Model}

The additive scoring system is listed in Table 4. A model score of 8 points corresponded to a risk of significant $\mathrm{CAD}$ of 0.05 . We recommend CAG screening at 8 or more points. The score for age was 2 points for every 5 years above 35 years, and hypertension and diabetes mellitus yielded a score of 2 points and 3 points, respectively. The score of angina was 13 points more than 8 points, suggesting it was an independent risk factor of coexistence CAD. Because any 
Table 1. Clinical Characteristics of the Study Population $(n=669)$

\section{Variable}

Age (years)

$51 \pm 9$

Male (\%)

$396(59.2 \%)$

Hypertension (\%)

$99(14.8 \%)$

Diabetes mellitus (\%)

$19(2.8 \%)$

Hypercholesterolemia (\%)

$17(2.5 \%)$

Family history of CAD (\%)

$22(3.3 \%)$

Ischemia changes on electrocardiogram (\%)

$48(7.2 \%)$

Smoking (\%)

$89(13.3 \%)$

CRP (mg/L)

$4.1 \pm 1.8$

NYHA class III or IV (\%)

$453(67.7 \%)$

Abnormal LV function (\%)

$583(87.1 \%)$

Atrial fibrillation (\%)

$175(26.2 \%)$

Angina (\%)

$49(7.3 \%)$

Concurrent MV replacement (\%)

$200(29.9 \%)$

Significant CAD (\%)

$88(13.2 \%)$

Concurrent CABG (\%)

$77(11.5 \%)$

Abbreviations: CAD, coronary artery disease; $C A B G$, coronary artery bypass graft; CRP, C-reactive protein; LV, left ventricular; MV, mitral valve; NYHA, New York Heart Association.

score more than 8 points would be an indication for $\mathrm{CAG}$ screening, the variable was reassigned a score of 8 points to simplify the scoring model system.

\section{Comparisons Between Logistic Model and Simple Additive Model}

The Hosmer-Lemeshow statistic revealed good fit with values of $6.3(P=0.6)$ and $7.0(P=0.5)$ for the logistic model and the simple additive model, respectively. The area under the ROC curve (AUC) of our logistic regression model was 0.948 , and the similar discriminating ability was achieved by the simple additive model with an AUC of 0.942 (Figure 1).

\section{Discussion}

Aortic valve replacement (AVR) is recommended as a standard surgical procedure for most patients with symptomatic aortic valve disease ${ }^{8}$ Concurrently combined with $\mathrm{CAD}$ as a risk factor for the prognosis of AVR, the independent predictors of impaired survival include advanced age, male sex, diabetes, noncongenital valvular pathology, or mixed valve disease, among others. ${ }^{9}$ It has been reported that concomitant $\mathrm{CABG}$ negates the effect of $\mathrm{CAD}$ on long-term survival. ${ }^{10,11}$ According to the AHA/ACC guidelines, ${ }^{2}$ concomitant revascularization is recommended in patients with aortic valve disease. Treatment of CAD by CABG at the time of AVR improves long-term survival with acceptable morbidity and mortality. ${ }^{12-14}$ It is of great
Table 2. Univariable Associations With Significant Coronary Artery Disease

\begin{tabular}{|c|c|c|c|c|}
\hline Variable & Coefficient & SE & OR & $P$ \\
\hline Age (years) ${ }^{a}$ & 0.882 & 0.090 & 2.416 & $<0.01$ \\
\hline Male (\%) & 0.758 & 0.257 & 2.135 & $<0.01$ \\
\hline Hypertension (\%) & 2.258 & 0.256 & 9.564 & $<0.01$ \\
\hline Diabetes mellitus (\%) & 1.410 & 0.490 & 4.098 & $<0.01$ \\
\hline Hypercholesterolemia (\%) & 1.596 & 0.507 & 4.935 & $<0.01$ \\
\hline Family history of CAD (\%) & -0.427 & 0.751 & 0.652 & 0.569 \\
\hline $\begin{array}{l}\text { Ischemia changes on } \\
\text { electrocardiogram (\%) }\end{array}$ & 0.030 & 0.226 & 1.030 & 0.997 \\
\hline Smoking (\%) & 0.141 & 0.325 & 1.152 & 0.663 \\
\hline High CRP (\%) & 0.563 & 0.299 & 1.755 & 0.06 \\
\hline NYHA class III or IV (\%) & 0.691 & 0.285 & 1.995 & 0.015 \\
\hline Abnormal LV function (\%) & 0.008 & 0.346 & 1.008 & 0.981 \\
\hline Atrial fibrillation (\%) & 0.258 & 0.250 & 1.295 & 0.301 \\
\hline Angina (\%) & 6.545 & 1.024 & 696.00 & $<0.01$ \\
\hline $\begin{array}{l}\text { Concurrent MV } \\
\text { replacement (\%) }\end{array}$ & -0.423 & 0.270 & 0.655 & 0.117 \\
\hline
\end{tabular}

Abbreviations: CAD, coronary artery disease; CRP, C-reactive protein; LV, left ventricular; MV, mitral valve; NYHA, New York Heart Association; $\mathrm{OR}$, odds ratio; SE, standard error. ${ }^{a}$ For every additional 5 years.

Table 3. ORs and Cls from the Original Regression Model and the Bootstrap Model

\begin{tabular}{|c|c|c|}
\hline Variable & $\begin{array}{l}\text { Original Model OR } \\
\qquad(95 \% \mathrm{Cl})\end{array}$ & $\begin{array}{l}\text { Bootstrap Model OR } \\
(95 \% \mathrm{Cl})\end{array}$ \\
\hline $\mathrm{Age}^{a}$ & $2.316(1.784-3.005)$ & $2.277(1.746-2.968)$ \\
\hline Hypertension & $4.134(1.939-8.817)$ & $3.412(1.557-7.477)$ \\
\hline $\begin{array}{l}\text { Diabetes } \\
\text { mellitus }\end{array}$ & $3.808(1.067-13.588)$ & $4.331(1.172-16.002)$ \\
\hline Angina & $745.35(82.785-6710.847)$ & $777.480(81.398-7426.166)$ \\
\hline
\end{tabular}

Table 4. A Simple Additive Scoring System for the Prediction of Significant CAD

\begin{tabular}{|lr|}
\hline Variable & Score $^{a}$ \\
\hline Age & 2 points for each 5 years over \\
\hline Hypertension & 2 points \\
\hline Diabetes mellitus & 3 points \\
\hline Angina & 8 points \\
\hline
\end{tabular}

Abbreviation: $\mathrm{CAD}$, coronary artery disease. ${ }^{a} \mathrm{~A}$ score equal to or more than 8 points is an indication for coronary angiography.

importance to screen with CAG in patients with high risk of coexistence of $\mathrm{CAD}$. The low specificity ${ }^{15}$ of the AHA/ACC 


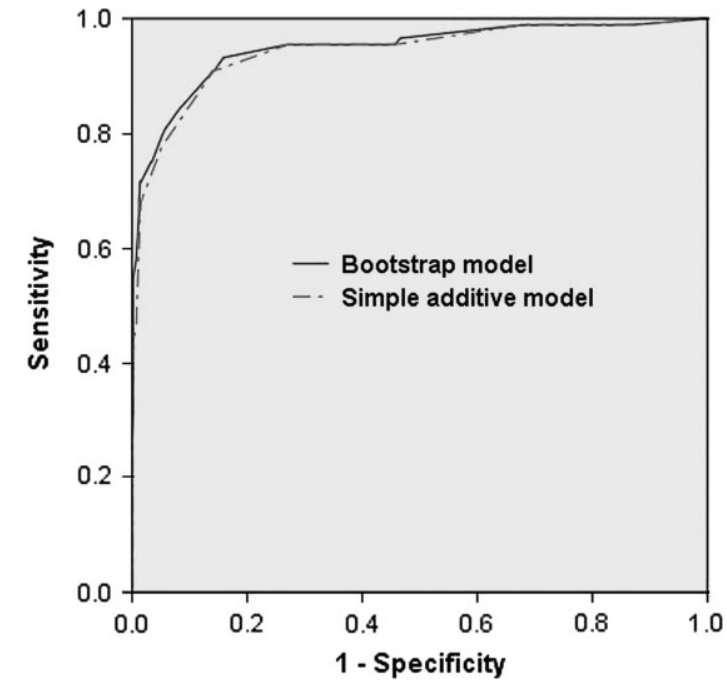

Figure 1. Comparison of ROC curves from the 2 models. The AUC of the bootstrap model and simple additive model were $0.948(95 \% \mathrm{Cl}$, $0.918-0.978)$ and 0.942 (95\% Cl, 0.911-0.973), respectively. Abbreviations: AUC, area under the ROC curve; $\mathrm{Cl}$, confidence interval; $\mathrm{ROC}$, receiver operating characteristic.

guidelines for CAG screening limited the wide use of the guidelines, especially for patients with rheumatic aortic valve disease in China and other developing countries, due to low incidence of $\mathrm{CAD}$, the cost, and possible the complications of CAG. If we applied the guidelines to our study population, we could find out that almost every patient should undergo CAG screening.

Several prior studies have concentrated on the predictive purely clinical models of significant $\mathrm{CAD}$ in symptomatic patients with chest pain. ${ }^{16-18}$ Based on established risk factor profiles, chest pain characteristics, and electrocardiographic criteria, Pryor et $\mathrm{al}^{17}$ developed and validated a model. Diamond and Forrester ${ }^{18}$ also built a model on the basis of age, sex, and nature of chest pain. These models excluded patients with typical or atypical angina, and applying these models to our patient population with rheumatic valve disease may be constrained by differences in baseline characteristics. To our knowledge, this is the first prediction model of significant $\mathrm{CAD}$ in patients undergoing operations for rheumatic aortic valve disease. We developed a logistic regression model and a simple additive model to better suit our population. In our study, both the models based on our rheumatic patients showed acceptable discriminative ability. With a probability of 0.05 as a cutoff, 325 patients were designated as having a low predicted risk for significant CAD. Of these patients, only 4 (1.2\%) patients had single-vessel disease, and none had high-risk CAD (ie, left main trunk, proximal left anterior descending, or multivessel disease). Significant CAD was present in 84 (24.4\%) of the 344 patients in the intermediate and high-risk groups.

There were 4 indicators of CAD in our model: age, angina, diabetes mellitus, and hypertension. Angina was reckoned as an independent indicator of significant $\mathrm{CAD}$, and all the patients with angina who undergo AVR with rheumatic heart valve disease should receive CAG screening before surgery. The result was similar to that of other studies. ${ }^{19-21}$ Green et $\mathrm{al}^{19}$ reported that about two-thirds of patients with severe aortic stenosis had angina pectoris, and that about one-half of these patients had CAD. Their study also found that $25 \%$ of the study patients without angina had angiographically significant CAD. In our study, $44(89.8 \%)$ of 49 patients with angina had significant $\mathrm{CAD}$. The prevalence of $\mathrm{CAD}$ in patients with angina in our rheumatic aortic population was much higher than that of prior studied populations. The result may be inferred by the size of the sample. It is necessary to expand the size of the sample in further studies to confirm the result. In some prior studies, angina was a less specific indicator of $\mathrm{CAD}$ in patients with valvular heart disease than in the general population because of increased wall stress or wall thickening with subendocardial ischemia, ${ }^{22}$ right ventricular (RV) chamber enlargement or hypertrophy, ${ }^{23}$ and others, whereas some commonly considered risk factors, such as family history, gender, hypercholesterolemia, and smoking were not predictive in our study. Kruczan et $\mathrm{al}^{4}$ inferred that the difference could be due to the demographic and clinical characteristics. But the accurate reason was still unknown. We noted a high prevalence of single-vessel disease in our patients without angina but with significant $\mathrm{CAD}$, and it has been reported that the presence of angina has been shown to be related to multivessel disease ${ }^{24,25}$; therefore, it was not surprising that most patients with $\mathrm{CAD}$ but without angina had singlevessel disease. In some studies, CAG was performed only in patients with angina, but from the results of our study, the other $44(7.0 \%)$ of 629 patients without angina also presented significant $\mathrm{CAD}$. The absence of angina did not reliably exclude CAG screening.

Another useful approach to identify low-risk patients is computed tomography angiography (CTA). As a noninvasive option, the CTA could provide the clinician with a whole image of the coronary artery system, including the degree of narrowing of the major coronary artery. Several prior studies have reported that CTA could rule out the presence of significant $\mathrm{CAD}$ with acceptable sensitivity in patients undergoing valve surgery. ${ }^{26-29}$ However, the results of 16- or 64-slice CTA could be inferred by atrial fibrillation, which commonly occurred in patients with rheumatic valve disease. In our study population, 175 (26\%) patients had atrial fibrillation, and 27 of these had significant $\mathrm{CAD}$. As the technique continues to develop, a 320-slice or dual-source CT scanner might overcome the limitation, but the results should be tested with clinical trials. Because of the high cost, these instruments are not easily applied clinically, and there is still a strong demand for a simple prediction system for $\mathrm{CAD}$ in patients with rheumatic aortic disease.

\section{Limitations}

This retrospective study is based on a group of patients of a single center with rheumatic aortic valve disease. For that reason, the size of our study population was limited, and the results should be confirmed by a prospective, multicenter study. There are some differences between rheumatic valve disease and degenerative or calcific valve disease both in pathologic and clinical characteristics, the reason for the difference in indicators for $\mathrm{CAD}$ in these 2 types of disease still needs further studies. 


\section{Conclusion}

Based on the rheumatic aortic valve population, we established a logistic model for the successful prediction of significant $\mathrm{CAD}$ in patients undergoing operations for rheumatic aortic valve disease, and furthermore we developed a simple additive score system to identify lowrisk patients with simplicity and accuracy. Both the logistic model and the simple additive scoring system achieved acceptable sensitivity and specificity.

\section{References}

1. Copeland JG, Griepp RB, Stinson EB, et al. Long-term follow-up after isolated aortic valve replacement. J Thorac Cardiovasc Surg. 1977;74:875-889.

2. Bonow RO, Carabello BA, Kanu C, et al. ACC/AHA 2006 guidelines for the management of patients with valvular heart disease: a report of the American College of Cardiology/American Heart Association Task Force on Practice Guidelines (writing committee to revise the 1998 Guidelines for the Management of Patients With Valvular Heart Disease): developed in collaboration with the Society of Cardiovascular Anesthesiologists: endorsed by the Society for Cardiovascular Angiography and Interventions and the Society of Thoracic Surgeons. Circulation. 2006;114:e84-e231.

3. Jose VJ, Gupta SN, Joseph G, et al. Prevalence of coronary artery disease in patients with rheumatic heart disease in the current era. Indian Heart J. 2004;56:129-131.

4. Kruczan DD, Silva NA, Pereira Bde B, et al. Coronary artery disease in patients with rheumatic and non-rheumatic valvular heart disease treated at a public hospital in Rio de Janeiro. Arq Bras Cardiol. 2008;90:197-203.

5. Effron B, Tibshirani RJ. An Introduction to the Bootstrap. New York: Chapman and Hall; 1993.

6. Iwi G, Millard RK, Palmer AM, et al. Bootstrap resampling: a powerful method of assessing confidence intervals for doses from experimental data. Phys Med Biol. 1999;44:N55-N62.

7. Zucchini W. An introduction to model selection. J Math Psychol. 2000;44:41-61

8. Braunwald E. Aortic valve replacement: an update at the turn of the millennium. Eur Heart J. 2000;21:1032-1033.

9. Jones JM, Lovell D, Cran GW, et al. Impact of coronary artery bypass grafting on survival after aortic valve replacement. Interact Cardiovasc Thorac Surg. 2006;5:327-330.

10. Flameng WJ, Herijgers P, Szecsi J, et al. Determinants of early and late results of combined valve operations and coronary artery bypass grafting. Ann Thorac Surg. 1996;61:621-628.

11. He GW, Grunkemeier GL, Starr A. Aortic valve replacement in elderly patients: influence of concomitant coronary grafting on late survival. Ann Thorac Surg. 1996;61:1746-1751.

12. Jones M, Schofield PM, Brooks NH, et al. Aortic valve replacement with combined myocardial revascularisation. Br Heart J. 1989;62:9-15.

13. Lund O, Nielsen TT, Pilegaard HK, et al. The influence of coronary artery disease and bypass grafting on early and late survival after valve replacement for aortic stenosis. JThorac Cardiovasc Surg. 1990;100:327-337.

14. Richardson JV, Kouchoukos NT, Wright JO 3rd, et al. Combined aortic valve replacement and myocardial revascularization: results in 220 patients. Circulation. 1979;59:75-81.

15. Lim E, Ali ZA, Barlow CW, et al. A simple model to predict coronary disease in patients undergoing operation for mitral regurgitation. Ann Thorac Surg. 2003;75:1820-1825.

16. L'Italien GJ, Paul SD, Hendel RC, et al. Development and validation of a Bayesian model for perioperative cardiac risk assessment in a cohort of 1,081 vascular surgical candidates. J Am Coll Cardiol. 1996;27:779-786.

17. Pryor DB, Harrell FE Jr, Lee KL, et al. Estimating the likelihood of significant coronary artery disease. Am J Med. 1983;75:771-780.

18. Diamond GA, Forrester JS. Analysis of probability as an aid in the clinical diagnosis of coronary-artery disease. $N$ Engl J Med. 1979;300:1350-1358.

19. Green SJ, Pizzarello RA, Padmanabhan VT, et al. Relation of angina pectoris to coronary artery disease in aortic valve stenosis. Am J Cardiol. 1985;55:1063-1065.

20. Garcia Rubira JC, Romero Chacon D, Naranjo D, et al. [Prevalence of coronary disease in patients with aortic stenosis]. Rev Esp Cardiol. 1992;45:427-431.

21. Pathak R, Padmanabhan VT, Tortolani AJ, et al. Angina pectoris and coronary artery disease in isolated, severe aortic regurgitation. Am J Cardiol. 1986;57:649-651.

22. Bertrand ME, LaBlanche JM, Tilmant PY, et al. Coronary sinus blood flow at rest and during isometric exercise in patients with aortic valve disease. Mechanism of angina pectoris in presence of normal coronary arteries. Am J Cardiol. 1981;47:199-205.

23. Ross RS. Right ventricular hypertension as a cause of precordial pain. Am Heart J. 1961;61:134-135.

24. Froelicher VF Jr, Yanowitz FG, Thompson AJ, et al. The correlation of coronary angiography and the electrocardiographic response to maximal treadmill testing in 76 asymptomatic men. Circulation. 1973;48:597-604.

25. Weiner DA, Ryan TJ, McCabe $\mathrm{CH}$, et al. Exercise stress testing. Correlations among history of angina, ST-segment response and prevalence of coronary-artery disease in the Coronary Artery Surgery Study (CASS). N Engl J Med. 1979;301:230-235.

26. Manghat NE, Morgan-Hughes GJ, Broadley AJ, et al. 16-detector row computed tomographic coronary angiography in patients undergoing evaluation for aortic valve replacement: comparison with catheter angiography. Clin Radiol. 2006;61:749-757.

27. Gilard M, Cornily JC, Pennec PY, et al. Accuracy of multislice computed tomography in the preoperative assessment of coronary disease in patients with aortic valve stenosis. J Am Coll Cardiol. 2006;47:2020-2024.

28. Meijboom WB, Mollet NR, Van Mieghem CA, et al. Pre-operative computed tomography coronary angiography to detect significant coronary artery disease in patients referred for cardiac valve surgery. J Am Coll Cardiol. 2006;48:1658-1665.

29. Reant P, Brunot S, Lafitte S, et al. Predictive value of noninvasive coronary angiography with multidetector computed tomography to detect significant coronary stenosis before valve surgery. $\mathrm{Am}$ J Cardiol. 2006;97:1506-1510. 